\title{
A global optimization using interval arithmetic
}

\author{
Ismail Bin Mohd*
}

Jabatan Matematik, Fakulti Sains dan Teknologi, Kolej Universiti Sains dan Teknologi Malaysia, Mengabang Telipot, 21030 Kuala Terengganu

${ }^{*}$ To whom correspondence should be addressed. E-mail: ismailmd@kustem.edu.my

Received 12 November 2005

http://dx.doi.org/10.11113/mjfas.v2n1-2.17

\section{ABSTRACT}

In order to establish an algorithm for bounding the global minimizers of a twice continuously differentiable function $f: R^{n} \rightarrow R^{1}$ in a given box ( a compact interval in $R^{n}$ ) using interval arithmetic, which is superior in the sense of less time needed compared to other method ([2]), in this paper we will attempt to describe as much as possible the ideas which presents and fulfill the explicitly mentioned algorithm.

| Interval mathematics | Symmetric operator | Lagrange multiplier | Gauss algorithm |

\section{Introduction}

Hansen [2] has described an algorithm, $\mathrm{H}$, for bounding the global minimizers of a twice continuously differentiable function $f: R^{n} \rightarrow R^{1}$ in a box that is a parallelepiped with sides parallel to the coordinate axes. In the algorithm $\mathrm{H}$, an interval form of Newton's method for bounding critical points of $f$, together with monotonicity and convexity tests and a continually-updated upper bound on the least value of $f$ in the box are used to delete sub-boxes which cannot contain global minimizers of $\mathrm{f}$.

Robinson [14] has described a technique for bounding a Kuhn-Tucker (KT) point $z^{*}=\left(x^{*}{ }^{*}, u^{*}{ }^{*}, w^{* T}\right)^{\top}$ for the nonlinear programming problem

$$
\begin{aligned}
& \min f(x) \\
& \text { subject to } \quad c_{i}(x) \geq 0 \quad(i=1, \ldots, m), \\
& h_{j}(x)=0 \quad(j=1, \ldots, r),
\end{aligned}
$$

by using a result from Nickel [13] to obtain a computable interval-arithmetic test for the existence of a unique KT point $Z^{*}$ in a given box in $R^{n+m+r}$ centred about an estimate of $Z^{*}$ which is obtained by using any convenient algorithm. 
Shearer and Wolfe [15] have described some computable existence and uniqueness tests for solutions of systems of nonlinear algebraic equations, and have also described an improved form of the Krawczyk-Moore algorithm, KMSW [16], and an improved form of the Alefeld-Platzoder algorithm, MAP [17].

Ismail [6] has described how some of the ideas of Hansen [2], and Shearer [15, 16] have been used in an algorithm, HM, for computing and bounding the global minimizer(s) of a twice continuously differentiable function $f: R^{n} \rightarrow R^{1}$ in a box.

The purpose of this paper is to describe how some of the ideas of Hansen [2], Ismail [4, 5, 6, 7, 8, 9], Robinson [14], and Shearer and Wolfe [15][16[17] have been used in an algorithm named MI, for bounding the global minimizers of $f: R^{n} \rightarrow R^{1}$ in a given box.

\section{Notation}

An interval number denoted by $\underline{\mathbf{X}}$, is defined by

$$
\underline{x}=\left[x_{1}, x_{S}\right]=\left\{x \mid x_{1} \leq x \leq x_{S}\right\}
$$

where $X_{I}$ and $X_{S}$ are called infimum and supremum, respectively. An nx1 interval vector (a box)

$$
\underline{x}=\left(\underline{x}_{\mathrm{i}}\right)_{\mathrm{nx} 1} \in \mathrm{I}\left(\mathrm{R}^{\mathrm{n}}\right)
$$

has ith element

$$
\underline{x}_{i}=\left[x_{i l}, x_{i S}\right] \in l(R)
$$

An nxn interval matrix

$$
\underline{A}=\left(\underline{a}_{i j}\right)_{n \times n} \in \mathrm{l}\left(M\left(R^{n}\right)\right)
$$

has $(i, j)$ th element

$$
\underline{a}_{i j}=\left[a_{i j l}, a_{i j S}\right] \in l(R)
$$

The magnitude $|$.$| , width w($.$) , and midpoint m($.$) mappings for \underline{x} \in \mathrm{l}(\mathrm{R})$ are defined by

$$
|\underline{x}|=\max \left\{\left|x_{1}\right|,\left|x_{S}\right|\right\}, w(\underline{x})=x_{S}-x_{1} \text {, and } m(\underline{x})=\frac{x_{1}+x_{S}}{2}
$$

respectively; for $\underline{x} \in I\left(R^{n}\right)$ they are defined by 


$$
|\underline{x}|=\left(|\underline{x}|_{i}\right)_{n x 1}, w(\underline{x})=\left(w\left(\underline{x}_{i}\right)\right)_{n \times 1} \text {, and } m(\underline{x})=\left(m\left(\underline{x}_{i}\right)\right)_{n \times 1}
$$

respectively; and for $\underline{A} \in \mathrm{I}\left(\mathrm{M}\left(\mathrm{R}^{\mathrm{n}}\right)\right)$ they are defined by

$$
|\underline{A}|=\left(\left|\underline{a}_{i j}\right|\right)_{n \times n}, w(\underline{A})=\left(w\left(\underline{a}_{i j}\right)\right)_{n x n} \text {, and } m(\underline{A})=\left(m\left(\underline{a}_{i j}\right)\right)_{n x n} \text {, }
$$

respectively.

The norm mappings $\|$. $\|$ are defined by

and

$$
\|\underline{x}\|=\max _{1 \leq i \leq n}\left\{\left|\underline{x}_{i}\right|\right\} \quad\left(\underline{x} \in I\left(R^{n}\right)\right)
$$

$$
\|\underline{A}\|=\max _{1 \leq i \leq n}\left(\sum_{j=1}^{n}\left|\underline{a}_{i j}\right|\right) \quad\left(\underline{A} \in I\left(M\left(R^{n}\right)\right)\right)
$$

If $V \subseteq R^{n}$ and $M \subseteq M\left(R^{n}\right)$ are given sets of nx1 real vectors and nxn real matrices respectively, then $\mathrm{I}(\mathrm{V})=\left\{\underline{\mathrm{x}} \in \mathrm{I}\left(\mathrm{R}^{\mathrm{n}}\right) \mid \underline{\mathrm{x}} \subseteq \mathrm{V}\right\}$, and $\mathrm{I}(\mathrm{M})=\left\{\underline{\mathrm{A}} \in \mathrm{I}\left(\mathrm{M}\left(\mathrm{R}^{\mathrm{n}}\right)\right) \mid \underline{\mathrm{A}} \subseteq \mathrm{M}\right\}$.

The sets $R^{n}$ and $M\left(R^{n}\right)$ are partially ordered through $(x \leq y) \Leftrightarrow\left(x_{i} \leq y_{i}(i=1, \ldots, n)\right)$ and $(A \leq B) \Leftrightarrow\left(a_{i j} \leq b_{i j}(i, j=1, \ldots, n)\right)$ respectively.

\section{The Global Optimization Problem}

Let $f: D \subseteq R^{n} \rightarrow R^{1}$ be a given function with $f \in C^{2}(\hat{D})$ where $\hat{D} \subset D$ is an open convex set. Let $\underline{\hat{x}} \in \mathrm{I}(\hat{D})$ be a given box. Let the nonlinear programming problem $P$ be defined by

$$
\left.\begin{array}{ll}
\min \quad f(x) \\
\text { subject to } \quad c_{i}(x) \geq 0 \quad(i=1, \ldots, 2 n)
\end{array}\right\} \quad \text { (problem P) }
$$

Where

$$
c_{i}(x)=x_{i}-\hat{x}_{i l} \quad(i=1, \ldots, n)
$$

and

$$
c_{i}(x)=\hat{x}_{i-n S}-x_{i-n} \quad(i=n+1, \ldots, 2 n) .
$$


A global minimizer $x^{*}$ of $f$ in $\underline{\hat{x}}$ is a solution of problem $P$ with Lagrange multipliers $u_{i}^{*}(i=1, \ldots, 2 n)$ such that

$$
\begin{aligned}
& F\left(z^{*}\right)=0 \\
& c\left(x^{*}\right) \geq 0 \\
& u^{*} \geq 0
\end{aligned}
$$

and

$$
f\left(x^{*}\right) \leq f(x) \quad(\forall x \in \underline{\hat{x}})
$$

where $F: R^{3 n} \rightarrow R^{3 n}$ is defined by

$$
F(z)=\left[\begin{array}{c}
\left(f^{\prime}(x)-u^{\top} c^{\prime}(x)\right)^{\top} \\
u_{1} c_{1}(x) \\
\vdots \\
u_{2 n} c_{2 n}(x)
\end{array}\right]
$$

in which

$$
z=\left(x^{\top}, u^{\top}\right)^{\top}, f^{\prime}(x)=\left(\partial_{i} f(x)\right)_{1 x n} \text {, and } c^{\prime}(x)=\left(\partial_{j} c_{i}(x)\right)_{2 n x n} .
$$

The global optimization problem which is solved by MI is equivalent to determining $z^{*} \in R^{3 n}$ such that (3.3) - (3.6) hold.

\section{Bounding the Lagrange Multipliers}

Given a box $\underline{\hat{z}}=\left(\underline{\hat{x}}^{\top}, \underline{\hat{u}}^{\top}\right)^{\top}$ containing the KT points for problem $P$, the algorithm MI deletes sub-boxes of $\underline{\hat{z}}$ which do not contain KT points corresponding to global minimizers of $f$ in $\underline{\hat{x}}$.

Initially, $\underline{\hat{u}} \in \mathrm{I}\left(\mathrm{R}^{2 \mathrm{n}}\right)$ must be determined. Let $\underline{f}^{\prime}: \mathrm{I}(\hat{\mathrm{D}}) \rightarrow \mathrm{I}\left(\mathrm{R}^{\mathrm{n}}\right)$ be a continuous inclusion monotonic interval extension of $f^{\prime}: \hat{D} \rightarrow R^{n}$, and let $\underline{\hat{d}}=\underline{f}^{\prime}(\underline{\hat{x}})^{\top}$. Then by (3.1) - (3.3), (3.5), and (3.7), for $\dot{i}=1, \ldots, n$, we obtain (for detail see [5])

$$
\begin{array}{ll}
\underline{\hat{u}}_{\mathrm{i}}=\underline{0} & \left(\hat{\mathrm{d}}_{\mathrm{iS}} \leq 0\right) \\
\underline{\hat{\mathrm{u}}}_{\mathrm{i}}=\left[0, \hat{\mathrm{d}}_{\mathrm{iS}}\right] & \left(\hat{\mathrm{d}}_{\mathrm{il}}<0<\hat{\mathrm{d}}_{\mathrm{iS}}\right)
\end{array}
$$




$$
\begin{array}{ll}
\underline{\hat{u}}_{i}=\underline{\mathrm{d}}_{\mathrm{i}} & \left(0 \leq \hat{\mathrm{d}}_{\mathrm{il}}\right) \\
\underline{\hat{\mathrm{u}}}_{\mathrm{i}+\mathrm{n}}=-\underline{\mathrm{d}}_{\mathrm{i}} & \left(\hat{\mathrm{d}}_{\mathrm{iS}} \leq 0\right) \\
\underline{\hat{\mathrm{u}}}_{\mathrm{i}+\mathrm{n}}=\left[0,-\hat{\mathrm{d}}_{\mathrm{il}}\right] & \left(\hat{\mathrm{d}}_{\mathrm{il}}<0<\hat{\mathrm{d}}_{\mathrm{iS}}\right) \\
\underline{\hat{\mathrm{u}}}_{\mathrm{i}+\mathrm{n}}=\underline{0} & \left(0 \leq \hat{\mathrm{d}}_{\mathrm{il}}\right)
\end{array}
$$

The formulae (3.8) - (3.13) are used in MI to determine $\underline{\hat{u}}$.

\section{Constructing Sub-boxes Which Might Contain KT Points}

If each side of a box is divided into two parts, then this could give rise to $2^{n}$ sub-boxes. Therefore, in order to prevent the generation of too many sub-boxes, Hansen [2] has suggested that only one side of the box with largest width is divided into two parts.

In [5], since we can avoid the disadvantage mentioned by Hansen [2], Ismail has shown how to derive a method for obtaining and computing $2^{n}$ sub-boxes $\underline{\hat{z}}^{(i)}\left(i=0, \ldots, 2^{n}-1\right)$ of $\underline{\hat{z}}$ which might contain the KT point

$$
z^{*}=\left(x^{* \top}, u^{* \top}\right)^{\top} \in \underline{\hat{z}} \text { for problem } P
$$

As explained in [5], let $b=\left(b_{j}\right)_{1 \times n}$ be the n-digit binary integer corresponding to the decimal integer $i$. For $(j=1, \ldots, n)$, if $b_{j}=1$, then

$$
\underline{\hat{x}}_{\mathrm{j}}^{(\mathrm{i})}=\left[\hat{\mathbf{x}}_{\mathrm{jl}}, \mathrm{m}\left(\underline{\hat{\mathbf{x}}}_{\mathrm{j}}\right)\right], \quad \underline{\hat{u}}_{\mathrm{j}}^{(\mathrm{i})}=\underline{\hat{u}}_{\mathrm{j}}, \text { and } \hat{\underline{u}}_{\mathrm{n}+\mathrm{j}}^{(\mathrm{i})}=\underline{0},
$$

and if $b_{j}=0$, then

$$
\underline{\hat{x}}_{j}^{(i)}=\left[m\left(\underline{\hat{x}}_{j}\right), \hat{\mathrm{x}}_{j S}\right], \quad \underline{\hat{u}}_{j}^{(i)}=\underline{0} \quad \text { and } \underline{\hat{u}}_{n+j}^{(i)}=\underline{\hat{u}}_{n+j} .
$$

\section{Ideas due to Hansen}

The monotonicity and convexity tests which are described in Section 9 and 5 of [2] , and HM [6] respectively, are used in MI.

The interval form of Hansen's method which is used in H [2], HM [6], and also in MI has the form

$$
\underline{y}_{k}=x_{k}-\left(\underline{a}_{k k}\right)^{-1}\left\{b_{k}+\sum_{j=1}^{k-1} \underline{a}_{k j}\left(\underline{x}_{j}^{\prime}-x_{j}\right)+\sum_{j=k+1}^{n} \underline{a}_{k j}\left(\underline{x}_{j}-x_{j}\right)\right\}
$$




$$
\underline{x}_{k}^{\prime}=\underline{y}_{k} \cap \underline{x}_{k}, k \in\{1, \ldots, n\}
$$

where $\underline{\mathrm{A}}=\left(\underline{\mathrm{a}}_{\mathrm{ij}}\right)_{\mathrm{nxn}}=\{\mathrm{m}(\underline{\mathrm{J}})\}^{-1} \underline{\mathrm{J}}, \mathrm{b}=\{\mathrm{m}(\underline{\mathrm{J}})\}^{-1}\left\{\mathrm{f}^{\prime}(\mathrm{m}(\underline{\mathrm{x}}))\right\}^{\top}$, and $\mathrm{x}_{\mathrm{k}}=\mathrm{m}\left(\underline{\mathrm{x}}_{\mathrm{k}}\right), \mathrm{k}=1, \ldots, \mathrm{n}$.

If $0 \notin \underline{a}_{k k}$, then $\underline{y}_{k}$ consists of one interval and hence $\underline{x}_{k}^{\prime}$ consists of at most one interval provided that $\underline{x}_{k}^{\prime} \neq \varnothing$; but if $0 \in \underline{a}_{k k}$, then $\underline{y}_{k}$ consists of at most two intervals say $\underline{y}_{k}^{(1)}$ and $\underline{y}_{k}^{(2)}$ ([5]). Therefore, Hansen [2] uses (6.1) and (6.2) for the cases $0 \notin \underline{a}_{k k}$ and $0 \in \underline{a}_{k k}$, dealt with separately.

A degenerate interval $\bar{f}$ such that $f^{*} \leq \bar{f}_{S}$ where $f^{*}$ is the globally minimum value of $f$ in $\underline{\hat{x}}$, is determined initially from $\underline{\bar{f}}=\underline{f}(m(\underline{\hat{x}}))$, where $\underline{f}: I(D) \rightarrow l(R)$ is a continuous inclusion monotonic interval extension of $f: D \rightarrow R$ and is updated at several points in MI. The interval $\underline{f}$ is used to delete sub-boxes $\underline{x}$ of $\underline{\hat{x}}$ such that $f(x)>f^{*}(\forall x \in \underline{x})$ using, in particular, the so-called quadratic method which is described in Section 7 of [2].

\section{The Symmetric Operator Test}

Let $\underline{\bar{z}}=\left(\underline{\bar{x}}^{\top}, \underline{\bar{u}}^{\top}\right)^{\top} \subseteq \underline{\hat{z}}$ and suppose that $\underline{z}=\left(\underline{z}_{\mathrm{i}}\right)_{\mathrm{tx} 1}(\mathrm{n} \leq \mathrm{t} \leq 3 \mathrm{n})$ is obtained from $\underline{\bar{z}}$ by deleting the zero intervals $\underline{\bar{Z}}_{i}$ corresponding to the zero Lagrange multiplier intervals from the last 2 n elements of $\overline{\bar{z}}$.

Let $F^{\prime}: I\left(R^{t}\right) \rightarrow I\left(M\left(R^{t}\right)\right)$ be a continuous inclusion monotonic interval extension of $F^{\prime}: R^{t} \rightarrow M\left(R^{t}\right)$ where $F: R^{t} \rightarrow R^{t}$ is defined by (3.7), and let

$$
\begin{aligned}
& A=\left(a_{i j}\right)_{t x t}=\left\{m\left(\underline{F}^{\prime}(\underline{z})\right)\right\}^{-1}, \\
& \underline{R}=\left(\underline{r}_{i j}\right)_{t x t}=I-A \underline{F}^{\prime}(\underline{z}), \\
& z=m(\underline{z}), \\
& b=A F(z), \\
& \underline{K}(\underline{z})=z-b+\underline{R}(\underline{z}-z), \\
& \underline{H}_{i}(\underline{z})=z_{i}-b_{i}+\sum_{j=1}^{i-1} \underline{r}_{i j}\left(\underline{H}_{j}^{\prime}(\underline{z})-z_{j}\right)+\sum_{j=i}^{t} \underline{r}_{i j}\left(\underline{z}_{j}-z_{j}\right), \\
& \underline{H}_{i}^{\prime}(\underline{z})=\underline{H}_{i}(\underline{z}) \cap \underline{z}_{i} \quad(i=1, \ldots, t), \\
& \underline{S}_{i}(\underline{z})=z_{i}-b_{i}+\sum_{j=1}^{i} r_{i j}\left(\underline{H}_{j}^{\prime}(\underline{z})-z_{j}\right)+\sum_{j=i+1}^{t} r_{i j}\left(\underline{S}_{j}^{\prime}(\underline{z})-z_{j}\right), \\
& \underline{S}_{i}^{\prime}(\underline{z})=\underline{S}_{i}(\underline{z}) \cap \underline{H}_{i}^{\prime}(\underline{z}) \quad(i=t, \ldots, 1),
\end{aligned}
$$


where I is the identity matrix.

Then $\underline{\mathrm{K}}, \underline{\mathrm{H}}$, and $\underline{\mathrm{S}}$ are the Krawczyk, Hansen, and Symmetric operation respectively. The following theorems are proved in [15].

\section{Theorem 7.1.}

(i) $\underline{\mathrm{S}}(\underline{\mathrm{z}}) \subseteq \underline{\mathrm{H}}(\underline{\mathrm{z}}) \subseteq \underline{\mathrm{K}}(\underline{\mathrm{z}})$.

(ii) If $z^{*} \in \underline{z}$ and $F\left(z^{*}\right)=0$, then $z^{*} \in \underline{S}(\underline{z})$.

(iii) If $\underline{\mathrm{S}}(\underline{\mathrm{z}}) \neq \varnothing$ and $\underline{\mathrm{S}}(\underline{\mathrm{z}}) \subseteq \underline{\mathrm{z}}$, then $\exists \mathrm{z}^{*} \in \underline{\mathrm{S}}(\underline{\mathrm{z}})$ such that $\mathrm{F}\left(\mathrm{z}^{*}\right)=0$.

(iv) If $\underline{\mathrm{S}}(\underline{\mathrm{z}}) \neq \varnothing, \underline{\mathrm{S}}(\underline{\mathrm{z}}) \subseteq \underline{\mathrm{z}}$, and $w(\underline{S}(\underline{z}))<w\left(\underline{H^{\prime}}(\underline{z})\right)$, then $\exists \mathrm{z}^{*} \in \underline{\mathrm{S}}(\underline{\mathrm{z}}), \mathrm{F}\left(\mathrm{z}^{*}\right)=0$ and $\mathrm{z}^{*}$ is unique in $\underline{Z}$.

\section{Theorem 7.2.}

If (1) $\underline{\mathrm{S}}(\underline{z}) \neq \varnothing,(2) \underline{\mathrm{S}}(\underline{z}) \subseteq \underline{z},(3) \mathrm{w}(\underline{\mathrm{s}}(\underline{z}))<\mathrm{w}\left(\underline{H^{\prime}}(\underline{z})\right)$, (4) the sequence $\left(\mathrm{z}^{(\mathrm{k})}\right)$ is generated from

$$
\mathrm{z}^{(\mathrm{k}+1)}=\mathrm{z}^{(\mathrm{k})}-\mathrm{AF}\left(\mathrm{z}^{(\mathrm{k})}\right) \quad(\mathrm{k} \geq 0)
$$

with $\mathrm{z} \in \underline{\mathrm{S}}(\underline{\mathrm{z}})$ arbitrary, then $\mathrm{z}^{(\mathrm{k})} \in \underline{\mathrm{S}}(\underline{\mathrm{z}})(\forall \mathrm{k} \geq 0)$, and $\mathrm{z}^{(\mathrm{k})} \rightarrow \mathrm{z}^{*}(\mathrm{k} \rightarrow \infty)$ where $\mathrm{z}^{*}$ is the unique zero in $\underline{\mathbf{Z}} \cdot \bullet$

If $\underline{H^{\prime}}(\underline{z})=\varnothing$ or if $\underline{S}^{\prime}(\underline{z})=\varnothing$, then by Theorem 7.1(i) and (ii) there is no zero of $\mathrm{F}$ in $\underline{z}$, so $\underline{\bar{z}}$ may be deleted from $\underline{\hat{Z}}$.

If $\underline{z} \subseteq \underline{S}(\underline{z})$ then $\underline{\bar{z}}$ is bisected into $\underline{z}^{(1)}$ and $\underline{z}^{(2)}$ for subsequent use in MI. If $\underline{S}(\underline{z}) \subseteq \underline{z}$ and $w(\underline{s}(\underline{z}))<w\left(\underline{H^{\prime}}(\underline{z})\right)$ then $\underline{z}$ is replaced with $\underline{S}(\underline{z})$ because from Theorem 7.1(iv) $\underline{S}(\underline{z})$ contains the unique zero $z^{*}$ of $F$ in $\underline{z}$. Also, from Theorem 7.2, the sequence $\left(z^{(k)}\right)$ generated from (7.10) with $z^{(0)}=m(\underline{S}(\underline{z}))$ remains in $\underline{S}(\underline{z})$ and converges to $z^{*}$.

If also $\underline{\underline{S}}(\underline{z}) \subset \operatorname{int}(\underline{z})$ then $(7.10)$ is equivalent to

$$
x^{(k+1)}=x^{(k)}-\tilde{A} f^{\prime}\left(x^{(k)}\right)^{\top} \quad(k \geq 0)
$$

where $\tilde{\mathrm{A}}=\left(\mathrm{a}_{\mathrm{ij}}\right)_{\mathrm{nxn}}$, and $\mathrm{x}^{(0)}=\left(\mathrm{m}(\underline{\mathrm{S}}(\underline{\mathrm{z}}))_{\mathrm{i}}\right)_{\mathrm{nx} \text { l }}$. The procedure (7.11) may be used to update $\overline{\mathrm{f}}$ by iterating until, for some $k \geq 1$,

$$
\left|\underline{f}\left(x^{(k)}\right)-\underline{f}\left(x^{(k-1)}\right)\right| \leq \varepsilon_{2} \max \left\{\left|\underline{f}\left(x^{(k)}\right)\right|, 1\right\}
$$


where $0<\varepsilon_{2}<1$, in which case $f$ has effectively ceased to change, or until for some $k \geq 2, f_{l}^{(k)}>f_{S}^{(k-1)}$ and $f_{1}^{(k-1)}>f_{S}^{(k-2)}$ where $\underline{f}^{(k)}=\underline{f}\left(x^{(k)}\right)(k \geq 0)$, in which case $z^{*}$ probably corresponds to a maximizer or to a saddle point of $\mathrm{f}$. If $\mathrm{X}^{(\mathrm{k})}$ satisfies (7.12) and $\mathrm{f}_{\mathrm{S}}^{(\mathrm{k})}<\overline{\mathrm{f}}_{\mathrm{I}}$ then $\underline{\mathrm{f}}$ is replaced with $\left[\mathrm{f}_{\mathrm{S}}^{(\mathrm{k})}, \mathrm{f}_{\mathrm{S}}^{(\mathrm{k})}\right]$.

If $\underline{\mathrm{S}}(\underline{\mathrm{z}}) \subseteq \underline{z}$ but $\mathrm{w}(\underline{\mathrm{s}}(\underline{z})) \geq \mathrm{w}\left(\underline{\mathrm{H}^{\prime}}(\underline{\mathrm{z}})\right)$ then $\underline{z}$ is replaced with $\underline{\mathrm{S}}(\underline{z})$ because from Theorem 7.1 (ii), (iii) every zero of $F$ in $\underline{z}$ lies in $\underline{S}(\underline{z})$ and there is at least one zero of $F$ in $\underline{z}$.

If $\underline{S^{\prime}}(\underline{z}) \neq \varnothing$ then $\underline{z}$ is replaced with $\underline{S}^{\prime}(\underline{z})$ because from Theorem 7.1(ii) if $z^{*} \in \underline{z}$ and $F\left(z^{*}\right)=0$ then $z^{*} \in \underline{S}(\underline{z})$.

The preceding ideas constitute the Symmetric Operator Test, which is used in MI to delete sub-boxes of $\underline{\underline{z}}$ which do not contain KT points for problem P, and to update $\underline{\bar{f}}$. If A defined by (7.1) does not exist, then the Symmetric operator Test cannot be used, so $\underline{\bar{z}}$ is bisected into $\underline{z}^{(1)}$ and $\underline{z}^{(2)}$ for subsequent use in MI.

\section{Using KMSW and MAP to Bound KT Points}

Let $\underline{z} \in \mathrm{l}\left(\mathrm{R}^{\mathrm{t}}\right)$ and $\mathrm{z}^{*} \in \mathrm{R}^{\mathrm{t}}$ be as in the Symmetric Operator Test. It is shown in [16] that if $\underline{S}(\underline{z}) \subseteq \underline{z}$, $\mathrm{w}(\underline{\mathrm{s}}(\underline{\mathrm{z}}))<\mathrm{w}\left(\underline{\mathrm{H}^{\prime}}(\underline{\mathrm{z}})\right),\|\underline{\mathrm{R}}\|<1$, and the sequence $\left(\mathrm{z}^{(\mathrm{k})}\right)$ is generated from the algorithm KMSW [16] with $\underline{\mathrm{z}}^{(0)}=\underline{\mathrm{z}}$ then $\mathrm{z}^{*} \in \underline{\mathrm{z}}^{(\mathrm{k})}(\forall \mathrm{k} \geq 0)$ and $\underline{\underline{z}}^{(\mathrm{k})} \rightarrow \mathrm{z}^{*}(\mathrm{k} \rightarrow \infty)$ at least quadratically. Then KMSW may be used in MI to compute sharp bounds on KT points.

Suppose that $\underline{z} \in \mathrm{l}\left(\mathrm{R}^{\mathrm{t}}\right)$ and $\mathrm{z}^{*} \in \mathrm{R}^{\mathrm{t}}$ are as in the Symmetric Operator Test and let $\underline{G}=\underline{F}^{\prime}(\underline{z}), B=m(\underline{G})$, $\mathrm{z}=\mathrm{m}(\underline{\mathrm{z}})$, and

$$
\underline{\mathrm{K}}_{N}(\underline{z}, \underline{G}, B)=\mathrm{z}-\underline{g}(B,\{F(z)-(B-\underline{G})(\underline{z}-z)\})
$$

where $\underline{g}(A, \underline{b}) \in l\left(R^{t}\right)$ is the result of applying the Gauss algorithm to the linear system $A \underline{x}=\underline{b}$ in which $A \in M\left(R^{t}\right)$ and $\underline{b} \in l\left(R^{t}\right)$. It is shown in [17] that if $B$ is nonsingular, $\exists \alpha \in[0,1)$ such that $\mathrm{w}\left(\underline{K}_{\mathrm{N}}(\underline{z}, \underline{G}, \mathrm{~B})\right) \leq \alpha \mathrm{w}(\underline{z})$, and the sequence $\left(\underline{z}^{(k)}\right)$ is generated from the algorithm MAP [17] with $\underline{\mathrm{z}}^{(0)}=\underline{\mathrm{z}}$, then $\mathrm{z}^{*} \in \underline{\mathrm{z}}^{(\mathrm{k})}(\forall \mathrm{k} \geq 0)$ and $\underline{\mathrm{z}}^{(\mathrm{k})} \rightarrow \mathrm{z}^{*}(\mathrm{k} \rightarrow \infty)$.

Furthermore, if $\exists \lambda>0$ such that 


$$
\left\|w\left(\underline{F^{\prime}}(\underline{\tilde{z}})\right)\right\| \leq \lambda\|w(\underline{\tilde{z}})\| \quad(\forall \underline{\tilde{z}} \in \underline{z})
$$

then $\underline{z}^{(k)} \rightarrow z^{*}(k \rightarrow \infty)$ at least quadratically. Thus MAP may be used in MI with

$$
\alpha=\max _{1 \leq i \leq t}\left\{\frac{w\left(\underline{K}_{N}(\underline{z}, \underline{G}, B)_{i}\right.}{w\left(\underline{z}_{i}\right)}\right\}
$$

to compute arbitrarily sharp bounds on KT points.

\section{Bisection and Selection Rules}

Suppose that the Symmetric Operator Test cannot guarantee the existence of a KT point $z^{*}$ in $\underline{z}=$ $\left(\underline{\mathbf{x}}^{\top}, \underline{\mathbf{u}}{ }^{\top}\right)^{\top} \subseteq \underline{\hat{z}}$. Therefore $\underline{z}$ is bisected into $\underline{z}^{(1)}$ and $\underline{\underline{z}}^{(2)}$ along the coordinate direction $\mathbf{j} \in\{1, \ldots, \mathrm{n}\}$ so that

$$
\begin{aligned}
& \underline{z}_{i}^{(1)}=\underline{z}_{i}^{(2)}=\underline{x}_{i}(i=1, \ldots, n ; i \neq j), \\
& \underline{z}_{j}^{(1)}=\left[x_{j l}, m\left(\underline{x}_{j}\right)\right],
\end{aligned}
$$

and

$$
\underline{z}_{j}^{(2)}=\left[m\left(\underline{x}_{j}\right), x_{j S}\right]
$$

Also

$$
\begin{aligned}
& \underline{z}_{i}^{(1)}=\underline{z}_{i}^{(2)}=\underline{u}_{i-n}(i=n+1, \ldots, 3 n ; i \neq n+j, 2 n+j), \\
& \underline{z}_{n+j}^{(1)}=\underline{u}_{j}, \quad \underline{z}_{2 n+j}^{(1)}=\underline{0}
\end{aligned}
$$

and

$$
\underline{z}_{n+j}^{(2)}=\underline{0}, \underline{z}_{2 n+j}^{(2)}=\underline{u}_{n+j}
$$

since

$$
\left(x_{j}^{*} \in \underline{z}_{j}^{(1)}\right) \Rightarrow\left(c_{n+j}\left(x^{*}\right)>0\right) \Rightarrow\left(u_{n+j}^{*}=0\right)
$$

and 


$$
\left(x_{j}^{*} \in \underline{z}_{j}^{(2)}\right) \Rightarrow\left(c_{j}\left(x^{*}\right)>0\right) \Rightarrow\left(u_{j}^{*}=0\right)
$$

if $z^{*}=\left(x^{*}, u^{*} T\right)^{\top}$ is a KT point for problem $P$.

The value of $\mathbf{j}$, and which of $\underline{z}^{(1)}$ and $\underline{z}^{(2)}$ is to be processed next in MI are determined by using ideas from [10] and [12].

\section{A Sub-box Deletion Test}

Let $\quad \underline{z}^{(i)}=\left(\underline{x}^{(i) \top}, \underline{u}^{(i) \top}\right)^{\top} \quad(i=1,2)$ be sub-boxes of $\underline{z}=\left(\underline{x}^{\top}, \underline{u}^{\top}\right)^{\top} \subseteq \underline{\hat{z}}$ which are produced either from the strategy which is described in Section 7 of [2] or from the bisection rules which are mentioned in the preceding section of this paper. It is possible to delete at least one of $\underline{z}^{(i)}(i=1,2)$ as follows.

Let $\underline{f}^{(i)}=\underline{f}\left(\underline{x}^{(i)}\right)(i=1,2)$. If, for $i=\{1,2\}, \bar{f}_{S}<f_{1}^{(i)}$, then delete $\underline{z}^{(i)}$.

If $\mathrm{f}_{\mathrm{S}}^{(2)}<\mathrm{f}_{\mathrm{I}}^{(1)}$, then delete $\underline{\mathrm{z}}^{(1)}$.

If $\mathrm{f}_{\mathrm{S}}^{(1)}<\mathrm{f}_{\mathrm{I}}^{(2)}$, then delete $\underline{z}^{(2)}$.

If $f_{I}^{(2)} \leq f_{I}^{(1)}, f_{S}^{(2)} \leq f_{S}^{(1)}$ and $f\left(m\left(\underline{x}^{(2)}\right)\right)<f_{I}^{(1)}$, then delete $\underline{z}^{(1)}$.

If $f_{I}^{(1)} \leq f_{I}^{(2)}, f_{S}^{(1)} \leq f_{S}^{(2)}$ and $f\left(m\left(\underline{x}^{(1)}\right)\right)<f_{I}^{(2)}$, then delete $\underline{z}^{(2)}$.

These tests have also been used by Ichida and Fujii [3].

\section{A Strict Complementary Slackness Test}

Suppose that $\underline{z}=\left(\underline{\mathbf{x}}^{\top}, \underline{u}^{\top}\right)^{\top} \subseteq \underline{\hat{z}}$ and $\exists \mathrm{z}^{*} \in \underline{\mathrm{z}}$ such that $\mathrm{F}\left(\mathrm{z}^{*}\right)=0$ and $0 \notin \underline{\mathrm{c}}_{\mathrm{i}} \cap \underline{u}_{\mathrm{i}} \quad(\mathrm{i}=1, \ldots, 2 \mathrm{n})$, where $\underline{c}_{i}=\underline{c}_{i}(\underline{x})(i=1, \ldots, 2 n)$. Then from $([4][14])$, strict complementary slackness holds at $z^{*}$. On the other hand, $0 \notin \underline{\mathrm{c}}_{\mathrm{i}} \cap \underline{\mathrm{u}}_{\mathrm{i}}(\mathrm{i}=1, \ldots, 2 \mathrm{n})$ is not necessary for strict complementary slackness.

If, however, it is known that $x^{*} \in \operatorname{int}(\underline{\hat{x}})$ where $z^{*}=\left(x^{*}, u^{*} T\right)^{\top}$, and $z^{*} \in \underline{z} \subset \operatorname{int}(\underline{\hat{z}})$ then $\mathrm{c}_{\mathrm{i}}(\mathrm{x})>0(\forall \mathrm{x} \in \underline{\mathrm{x}})(\mathrm{i}=1, \ldots, 2 \mathrm{n})$ whence $0 \notin \underline{\mathrm{c}}_{\mathrm{i}} \cap \underline{\mathrm{u}}_{\mathrm{i}}(\mathrm{i}=1, \ldots, 2 \mathrm{n})$.

This suggest that it might be beneficial to bisect $\underline{z}$ if for some $i \in\{1, \ldots, 2 n\}, 0 \in \underline{\mathbf{C}}_{i} \cap \underline{u}_{i}$ when it is known that $x^{*} \in \operatorname{int}(\underline{\hat{x}})$. 
If $x^{*} \in \partial(\underline{\hat{x}})$, so that for at least one $i \in\{1, \ldots, 2 n\}, x_{i}^{*}=\hat{x}_{i l}$ or $x_{i}^{*}=\hat{x}_{i S}$ then $c_{i}\left(x^{*}\right)=0$ so if $z^{*} \in \underline{z}$ then $0 \in \underline{\mathbf{c}}_{\mathrm{i}}$. In this case bisection might not be beneficial because it could occur an indefinite number of times and produce an excessively large number of boxes.

In practice, however, it is found to be desirable to check whether $0 \notin \underline{\mathrm{C}}_{\mathrm{i}} \cap \underline{\mathrm{u}}_{\mathrm{i}}(\mathrm{i}=1, \ldots, 2 \mathrm{n})$ even when it is not known that $\mathrm{X}^{*} \in \operatorname{int}(\underline{\hat{\mathrm{x}}})$, and to avoid the excessively large number of bisections which could occur by bisecting along the coordinate direction $\mathrm{j}$ only if $\mathrm{w}\left(\underline{x}_{\mathrm{j}}\right)>\varepsilon_{0}$ where $\varepsilon_{0} \in(0,1)$ is such that if $\mathrm{x}^{*} \in \underline{\mathrm{X}}$ and $\|\mathrm{W}(\underline{\mathrm{x}})\| \leq \varepsilon_{0}$ then $\underline{\mathrm{X}}$ is a sufficiently sharp bound on $\mathrm{X}^{*}$.

\section{Numerical Examples}

The algorithm MI, which incorporates the ideas which are mentioned in the preceding sections, and the algorithm $\mathrm{H}$, have been implemented. Convergence is considered to have occurred when each global minimizer $\mathrm{X}^{*}$ is bounded within a box $\underline{x}$ such that $\|\mathrm{W}(\underline{\mathrm{x}})\| \leq 10^{-6}$ and the globally minimum value $\mathrm{f}^{*}$ of $\mathrm{f}$ is bounded within an interval $\underline{f}$ such that $w(\underline{f}) \leq 10^{-6}$.

The following examples illustrates the behaviour of MI.

Example 12.1

$$
\begin{aligned}
& f(x)=16\left(x_{1}+x_{2}\right)^{2}+\left\{4\left(x_{1}+x_{2}\right)+\left(x_{1}-x_{2}\right)\left(x_{1}-2\right)+x_{2}^{2}-1\right\}^{2} \\
& \underline{\hat{x}}=\left(\underline{\hat{x}}_{1}, \underline{\hat{x}}_{2}\right)^{\top}=([-2,4],[-2,4])^{\top}=([-2,4])_{2 x 1}
\end{aligned}
$$

Example 12.2

$$
\begin{aligned}
& f(x)=\left(x_{1}^{2}+x_{2}^{2}+x_{1} x_{2}\right)^{2}+\sin ^{2} x_{1}+\cos ^{2} x_{2} \\
& \underline{\hat{x}}=([-1,2])_{2 x 1}
\end{aligned}
$$

The function $\mathrm{f}$ has two global minimizers in the interior of $\underline{\hat{x}}$.

Example 12.3

$$
\begin{aligned}
& f(x)=\left(1-x_{1}\right)^{2}+\left(1-x_{n}\right)^{2}+\sum_{i=1}^{n-1}\left(x_{i}^{2}-x_{i+1}\right)^{2} \\
& \underline{\hat{x}}=([0.5,1])_{n x 1} \text { where } n \geq 2 .
\end{aligned}
$$


The function $\mathrm{f}$ has one global minimizer $\mathrm{x}^{*}=(1)_{\mathrm{nx} 1}$ on the boundary of $\underline{\hat{x}}$ and strict complementary slackness does not hold at $\mathrm{X}^{*}$.

\section{Discussion and Conclusion}

In the Triplex ([1][11]) implementations of both $\mathrm{H}$ and $\mathrm{MI}$ it is possible to ignore the boundary of $\underline{\hat{x}}$. This is useful if it is known that $\mathrm{X}^{*} \in \operatorname{int}(\underline{\hat{x}})$ because it usually leads to less computational labour if it is known that the boundary of $\underline{\hat{x}}$ can be deleted.

Table 13.1 contains the CPU times, in seconds, corresponding to examples 12.1 and 12.2 when it is known that $\mathrm{x}^{*} \in \operatorname{int}(\underline{\hat{x}})$, and when it is known only that $\mathrm{x}^{*} \in \underline{\hat{\mathrm{x}}}$.

Table 13.1 : Computational time in seconds

\begin{tabular}{|c|c|c|c|c|}
\hline Example & \multicolumn{2}{|c|}{$\mathrm{X}^{*} \in \operatorname{int}(\underline{\hat{\mathbf{x}}})$} & \multicolumn{2}{c|}{$\mathrm{X}^{*} \in \hat{\mathrm{x}}$} \\
\hline & $\mathrm{H}$ & $\mathrm{MI}$ & $\mathrm{H}$ & MI \\
\hline 12.1 & 130.1 & 99.70 & 133.0 & 106.0 \\
12.2 & 821.0 & 459.0 & 811.0 & 524.0 \\
\hline
\end{tabular}

For Example 12.2, the CPU time required by $\mathrm{H}$ when $\mathrm{X}^{*} \in \operatorname{int}(\underline{\hat{x}})$ is slightly larger than that which is required when $x^{*} \in \underline{\hat{x}}$ because 20 more Hessian diagonal evaluations are needed when $x^{*} \in \operatorname{int}(\underline{\hat{x}})$.

Table 13.2 contains the CPU times, in seconds, corresponding to Example 12.3 for various values of $n$.

Table 13.2 : Computational time in seconds

\begin{tabular}{|c|c|c|c|c|}
\hline $\mathrm{n}$ & \multicolumn{2}{|c|}{$\mathrm{x}^{*} \in \operatorname{int}(\underline{\hat{\mathrm{x}}})$} & \multicolumn{2}{c|}{$\mathrm{X}^{*} \in \hat{\mathrm{x}}$} \\
\hline & $\mathrm{H}$ & MI & H & MI \\
\hline 2 & 5.98 & 7.72 & 66.6 & 66.2 \\
3 & 19.9 & 22.5 & 298.0 & 308.0 \\
4 & 47.1 & 42.1 & 748.0 & 893.0 \\
5 & 125.0 & 73.0 & 2820.0 & 2080.0 \\
\hline
\end{tabular}

For Example 12.3 for all values of $\mathrm{n}$, both $\mathrm{H}$ and $\mathrm{MI}$ are able to bound the global minimizer even when it is assumed that $\mathrm{x}^{*} \in \underline{\hat{\mathbf{x}}}$.

Computational experience with the Triplex implementations of $\mathrm{H}$ and MI indicates that, with few exceptions, MI requires fewer evaluations of $f, f^{\prime}$, and $f^{\prime \prime}$ than does H. This might account for the increasing superiority of MI over $\mathrm{H}$ as $\mathrm{n}$ increases in Example 12.3, as shown in Table 13.2. 


\section{References}

[1] Bailey, P. J., Cole, A. J., and Morrison, R., Triplex User Manual, University of St. Andrews Department of Computational Science Report CS/82/5, 1982.

[2] Hansen, E. R., Numerische Mathematik 34 (1980) 247 - 270.

[3] Ichida, K., and Fujii, Y., Computing 23 (1979) 85 - 97.

[4] Ismail, B. M., Applied Mathematics and Computation, 1989

[5] Ismail, B. M., Pertanika 13(3) (1990) 409 -413.

[6] Ismail, B. M., Computational and Applied Mathematics, 31(1990) $373-382$.

[7] Ismail, B. M., Asean Journal on Science and Technology for Development, 7(2)(1990) 1 - 11.

[8] Ismail, B. M., Computational and Applied Mathematics, 58(1995) $183-192$.

[9] Ismail, B. M., Applied Mathematics and Computation, 110(2000) 121 - 131.

[10] Jones, S. T., Searching for Solutions of Finite Nonlinear Systems - An Interval Approach, Ph. D. Thesis, University of Winsconsin, Madison U.S.A., 1978.

[11] Morrison, R., Cole, A. J., Bailey, P. J., Wolfe, M. A., and Shearer, J. M., Experience in Using a High Level Language that Supports Interval Arithmetic, In proceedings of ARITH6, the Joint TCCA/IEEE Sixth Symposium on Computer Arithmetic, Aarhus, Denmark, 1983.

[12] Moore, R. E., and Jones, S. T., SIAM Journal on Numerical Analysis, 14(1977) $1051-1065$.

[13] Nickel, K. L. E., On the Newton Method in Interval Analysis, MRC Technical Summary Report 1136 (1971) Madison, Wisconsin, U. S. A.

[14] Robinson, S. M., Mathematical Programming, 5 (1973) 235 - 242.

[15] Shearer, J. M., and Wolfe, M. A., SIAM Journal on Numerical Analysis 22(1985) $1200-1207$.

[16] Shearer, J. M., and Wolfe, M. A., Applied Mathematics and Computation 17(1985) 229 - 239.

[17] Shearer, J. M., and Wolfe, M. A., SIAM Journal on Scientific and Statistical Computing, 1986. 